\title{
Quantum Energy-Transport and Drift-Diffusion Models for Electron Transport in Graphene An Approach by The Wigner Function
}

\section{Vito Dario Camiola ( $\sim$ dario.camiola@unict.it )}

Università degli Studi di Catania: Universita degli Studi di Catania https://orcid.org/0000-0001-73828499

\section{Giovanni Mascali}

Universita della Calabria

\section{Vittorio Romano}

Università degli Studi di Catania: Universita degli Studi di Catania

\section{Research Article}

Keywords: Wigner equation, Graphene, Wigner equilibrium function

Posted Date: July 12th, 2021

DOI: https://doi.org/10.21203/rs.3.rs-580204/v1

License: (c) (i) This work is licensed under a Creative Commons Attribution 4.0 International License. Read Full License

Version of Record: A version of this preprint was published at Journal of Computational Electronics on November 11th, 2021. See the published version at https://doi.org/10.1007/s10825-021-01782-9. 


\title{
Quantum energy-transport and drift-diffusion models for electron transport in graphene
}

\section{An approach by the Wigner function}

\author{
Vito Dario Camiola · Giovanni Mascali . \\ Vittorio Romano
}

Received: date / Accepted: date

\begin{abstract}
The present work aims at formulating quantum energy-transport and driftdiffusion equations for charge transport in graphene from a quantum hydrodynamic model proposed in [1], obtained from the Wigner-Boltzmann equation via the moment method. In analogy with the semiclassical case, we are confident that the energytransport and drift-diffusion models have mathematical properties which allow an easier numerical treatment.
\end{abstract}

Keywords Wigner equation - Graphene · Wigner equilibrium function

\section{Introduction}

Graphene, a monolayer of $s p^{2}$-bonded carbon atoms, is not only the basis for graphite but also a new material with immense potential in microelectronics for its exceptional electrical transport properties, like high conductivity and high charge mobility. As a result of its promising properties, it seems to be an ideal candidate to take over from silicon for the next generation of faster and smaller electronic devices.

If the length of the active area is of the order of few nanometers, quantum phenomena must be taken into account and a semiclassical description of charge transport is no longer adequate. The present work aims at formulating quantum energytransport and drift-diffusion models for a proper description of charge transport in

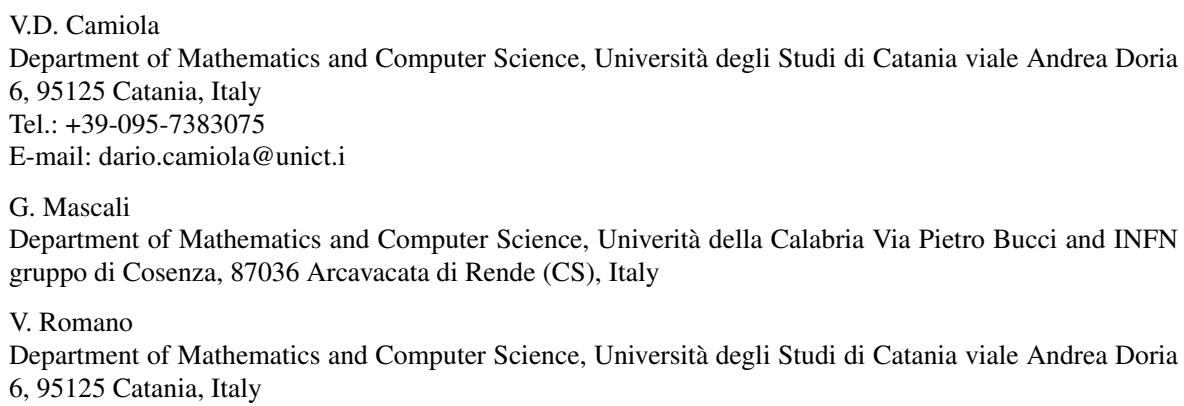


graphene, which is extremely important for the growing technological development in CAD tools.

In [1] a quantum hydrodynamic model for charge transport in graphene is derived from a moment expansion of the Wigner-Boltzmann equation and the needed closure relations are obtained by adding quantum corrections based on the equilibrium Wigner function to the semiclassical model formulated in [2-6] by exploiting the Maximum Entropy Principle. The expression of the equilibrium Wigner function which takes into account the form of the energy band of graphene has been obtained by solving the corresponding Bloch equation. In the present work, from this hydrodynamic model, a quantum energy-transport and a quantum drift-diffusion model are deduced in the long time asymptotic limit.

The plan of the paper is as follows. In section 1 the main features of the energy band in graphene are recalled. In section 2 the Wigner equation in graphene is introduced and a quantum hydrodynamic model is obtained as associated moment system. The last section is devoted to the deduction of a quantum energy-transport and a quantum drift-diffusion model.

\section{Band structure on graphene}

Graphene is a two-dimensional crystal made of carbon atoms arranged into a honeycomb lattice structure. Solving the one-electron Schröndinger equation including only the periodic potential due to the charges of the crystal in the tight-binding approximation yields the relation (see [7] for a comprehensive review on the topic)

$$
\mathscr{E}_{ \pm}(\mathbf{k})= \pm t \sqrt{3+f(\mathbf{k})}-t^{\prime} f(\mathbf{k})
$$

The function $f$ reads

$$
f(\mathbf{k})=4 \cos \left(\frac{3 a}{2} k_{x}\right) \cos \left(\frac{a \sqrt{3}}{2} k_{y}\right)+2 \cos \left(a \sqrt{3} k_{y}\right),
$$

$\mathbf{k}$ being the two dimensional electron quasi-wave vector with respect to the center of the first Brillouin zone. The sign + refers to the conduction band, while the sign refers to the valence band. The constant $a$ is the interatomic distance (of the order of one Angstrom); $t \approx 2.7 \mathrm{eV}$ is the hopping parameter between the first nearestneighbour orbitals, while $t^{\prime}$ is the hopping parameter between the second nearestneighbour orbitals and its value is still matter of debate. The function $3+f(\mathbf{k})$ is zero at the vertices, named Dirac points, of the Brillouin zone. By assuming $t^{\prime}=0$ and by choosing in the $\mathbf{k}$-space a reference frame centered in the considered Dirac point, for $|\mathbf{k}| a<<1$, that is near the Dirac points, one gets the following linear dispersion relation (see Fig. 1)

$$
\mathscr{E}_{ \pm}(p)= \pm v_{F} p
$$

where $\mathbf{p}=\hbar \mathbf{k}$ is the electron quasi-momentum with $\hbar$ the reduced Planck constant. A nonzero value of $t^{\prime}$ breaks the symmetry with respect to $\mathscr{E}=0$, and shifts the energy at the conical point from $\mathscr{E}=0$ to $\mathscr{E}=3 t^{\prime}$. 


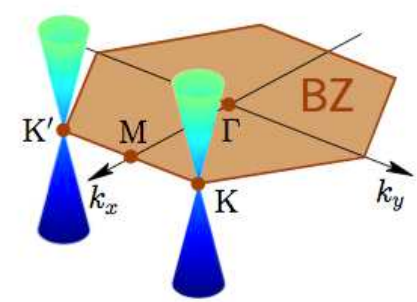

Fig. 1 Schematic representation of the energy band around the Dirac points K and K'.

In order to include the presence of a small gap between the conduction and the valence band, we assume $t^{\prime} \neq 0$ in the tight-binding approximation, therefore the Hamiltonian describing the energy in the proximity of the Dirac points can be written in the form

$$
H=v_{F}\left(\begin{array}{cc}
\alpha & p_{x}+i p_{y} \\
p_{x}-i p_{y} & -\alpha
\end{array}\right)
$$

which has eigenvalues

$$
\mathscr{E}_{ \pm}(\mathbf{p})= \pm v_{F} \sqrt{\alpha^{2}+p^{2}}
$$

where $\alpha$ is a small parameter related to the nearest-neighbour hopping energy. A similar regularization has been also adopted in [8] and can be also useful for describing charge behavior in double layer graphene and graphene nanoribbons.

\section{Wigner equation for charge transport in graphene}

From now on, for the sake of simplicity, we will consider only electrons in the conduction bands. The inclusion of holes can be done straightforwardly. A way to formulate a quantum transport description is that of introducing a single electron Wigner quasi-distribution $w(\mathbf{x}, \mathbf{p}, t)$, depending on the position $\mathbf{x}$, momentum $\mathbf{p}$ and time $t$. Its evolution is governed by the Wigner-Poisson system for $w$ and the electrostatic potential $\Phi$

$$
\begin{aligned}
& \frac{\partial w(\mathbf{x}, \mathbf{p}, t)}{\partial t}+S[\mathscr{E}] w(\mathbf{x}, \mathbf{p}, t)-q \theta[\mathscr{E}] w(\mathbf{x}, \mathbf{p}, t)=C[w], \\
& \nabla \cdot(\varepsilon \nabla \Phi)=-q\left(N_{D}-n\right),
\end{aligned}
$$

where $q$ is the elementary (positive) charge, $N_{D}$ is donor carrier concentration, $\varepsilon$ is the dielectric constant, $C[w]$ is the collision term representing the electron-phonon 
scattering while $S[\mathscr{E}]$ and $\theta[\mathscr{E}]$ represent the pseudo-differential operators

$$
\begin{array}{r}
S[\mathscr{E}] w(\mathbf{x}, \mathbf{p}, t)= \\
\frac{i}{\hbar(2 \pi)^{2}} \int_{\mathbb{R}_{\mathbf{x}^{\prime}}^{2} \times \mathbb{R}_{V}^{2}}\left[\mathscr{E}\left(\mathbf{p}+\frac{\hbar}{2} v, t\right)-\mathscr{E}\left(\mathbf{p}-\frac{\hbar}{2} v, t\right)\right] w\left(\mathbf{x}^{\prime}, \mathbf{p}, t\right) e^{-i\left(\mathbf{x}^{\prime}-\mathbf{x}\right) \cdot v} d \mathbf{x}^{\prime} d v, \\
\theta[\mathscr{E}] w(\mathbf{x}, \mathbf{p}, t)= \\
\frac{i}{\hbar(2 \pi)^{2}} \int_{\mathbb{R}_{\mathbf{p}^{\prime}}^{2} \times \mathbb{R}_{\eta}^{2}}\left[\Phi\left(\mathbf{x}+\frac{\hbar}{2} \eta, t\right)-\Phi\left(\mathbf{x}-\frac{\hbar}{2} \eta, t\right)\right] w\left(\mathbf{x}, \mathbf{p}^{\prime}, t\right) e^{i\left(\mathbf{p}^{\prime}-\mathbf{p}\right) \cdot \eta} d \mathbf{p}^{\prime} d \eta .
\end{array}
$$

In the semiclassical limit $\hbar \rightarrow 0$, the Wigner equation reduces to the semiclassical Boltzmann one. The direct numerical solution of the Wigner equation is a daunting computation task [9]. However, the appealing aspect of quantum transport in terms of the Wigner function is that one can define the macroscopic quantities of interest as expectation values (moments) like in the semiclassical case although the Wigner function is real but not defined in sign. For example, a 6-moment model can be formulate by considering the following moments which have a direct physical meaning

$$
\begin{aligned}
& \frac{4}{(2 \pi \hbar)^{2}} \int_{\mathbb{R}^{2}} w(\mathbf{x}, \mathbf{p}, t) d \mathbf{p}=n(\mathbf{x}, t) \quad \text { density, } \\
& \frac{4}{(2 \pi \hbar)^{2}} \int_{\mathbb{R}^{2}} w(\mathbf{x}, \mathbf{p}, t) \mathscr{E}(p) d \mathbf{p}=n(\mathbf{x}, t) W \quad \text { energy density, } \\
& \frac{4}{(2 \pi \hbar)^{2}} \int_{\mathbb{R}^{2}} w(\mathbf{x}, \mathbf{p}, t) \mathbf{v}(p) d \mathbf{p}=n(\mathbf{x}, t) \mathbf{V} \quad \text { linear momentum density, } \\
& \frac{4}{(2 \pi \hbar)^{2}} \int_{\mathbb{R}^{2}} w(\mathbf{x}, \mathbf{p}, t) \mathscr{E}(p) \mathbf{v}(p) d \mathbf{p}=n(\mathbf{x}, t) \mathbf{S} \quad \text { energy-flux density. }
\end{aligned}
$$

where the factor 4 takes into account the spin and valley degeneration.

The corresponding evolution equations are given by ${ }^{1}$

$$
\begin{gathered}
\frac{\partial}{\partial t} n(\mathbf{x}, t)+\frac{\partial}{\partial x_{i}}\left(n(\mathbf{x}, t) V_{i}-\frac{\hbar^{2}}{24} \frac{\partial^{2}\left(n(\mathbf{x}, t) T_{i j k}^{(0)}\right)}{\partial x_{j} \partial x_{k}}\right)=0, \\
\frac{\partial}{\partial t}(n(\mathbf{x}, t) W)+\frac{\partial}{\partial x_{i}}\left(n(\mathbf{x}, t) S_{i}-\frac{\hbar^{2}}{24} \frac{\partial^{2}\left(n(\mathbf{x}, t) T_{i j k}^{(1)}\right)}{\partial x_{j} \partial x_{k}}\right) \\
-q \frac{\partial}{\partial x_{i}} \Phi(\mathbf{x}) n(\mathbf{x}, t) V_{i}+\frac{q \hbar^{2}}{24} \frac{\partial^{3} \Phi(\mathbf{x})}{\partial x_{i} \partial x_{j} \partial x_{k}} n(\mathbf{x}, t) T_{i j k}^{(0)}=n C_{W}[w], \\
\frac{\partial}{\partial t}\left(n(\mathbf{x}, t) V_{i}\right)+\frac{\partial}{\partial x_{j}}\left(n(\mathbf{x}, t) F_{i j}^{(0)}-\frac{\hbar^{2}}{24} \frac{\partial^{2}\left(n(\mathbf{x}, t) H_{i j k l}^{(0)}\right)}{\partial x_{k} \partial x_{l}}\right) \\
-q \frac{\partial}{\partial x_{j}} \Phi(\mathbf{x}) n(\mathbf{x}, t) G_{i j}^{(0)}+\frac{q \hbar^{2}}{24} \frac{\partial^{3} \Phi(\mathbf{x})}{\partial x_{j} \partial x_{k} \partial x_{l}} n(\mathbf{x}, t) L_{i j k l}^{(0)}=n C_{V_{i}}[w],
\end{gathered}
$$

\footnotetext{
${ }^{1}$ Einstein's summation convention is used
} 


$$
\begin{aligned}
& \frac{\partial}{\partial t}\left(n(\mathbf{x}, t) S_{i}\right)+\frac{\partial}{\partial x_{j}}\left(n(\mathbf{x}, t) F_{i j}^{(1)}-\frac{\hbar^{2}}{24} \frac{\partial^{2}\left(n(\mathbf{x}, t) H_{i j k l}^{(1)}\right)}{\partial x_{k} \partial x_{l}}\right) \\
& -q \frac{\partial}{\partial x_{j}} \Phi(\mathbf{x}) n(\mathbf{x}, t) G_{i j}^{(1)}+\frac{q \hbar^{2}}{24} \frac{\partial^{3} \Phi(\mathbf{x})}{\partial x_{j} \partial x_{k} \partial x_{l}} n(\mathbf{x}, t) L_{i j k l}^{(1)}=n C_{S_{i}}[w]
\end{aligned}
$$

where $V_{i}$ and $S_{i}$ are the significant components of macroscopic velocity $\mathbf{V}$ and energyflux $\mathbf{S}$ respectively. Besides the average densities, velocities, energies and energy fluxes, additional quantities appear

$$
\begin{aligned}
n(\mathbf{x}, t)\left(\begin{array}{c}
F_{i j}^{(0)} \\
F_{i j}^{(1)}
\end{array}\right) & =\frac{4}{(2 \pi \hbar)^{2}} \int_{\mathbb{R}^{2}}\left(\begin{array}{c}
1 \\
\mathscr{E}(p)
\end{array}\right) w(\mathbf{x}, \mathbf{p}, t) v_{i} v_{j} d \mathbf{p}, \\
n(\mathbf{x}, t)\left(\begin{array}{c}
G_{i j}^{(0)} \\
G_{i j}^{(1)}
\end{array}\right) & =\frac{4}{(2 \pi \hbar)^{2}} \int_{\mathbb{R}^{2}}\left(\begin{array}{c}
1 \\
\mathscr{E}(p)
\end{array}\right) w(\mathbf{x}, \mathbf{p}, t) \frac{\partial^{2} \mathscr{E}(p)}{\partial p_{i} \partial p_{j}} d \mathbf{p}, \\
n(\mathbf{x}, t)\left(\begin{array}{c}
T_{i j k}^{(0)} \\
T_{i j k}^{(1)}
\end{array}\right) & =\frac{4}{(2 \pi \hbar)^{2}} \int_{\mathbb{R}^{2}}\left(\begin{array}{c}
1 \\
\mathscr{E}(p)
\end{array}\right) w(\mathbf{x}, \mathbf{p}, t) \frac{\partial^{3} \mathscr{E}(p)}{\partial p_{i} \partial p_{j} \partial p_{k}} d \mathbf{p}, \\
n(\mathbf{x}, t)\left(\begin{array}{c}
H_{i j k l}^{(0)} \\
H_{i j k l}^{(1)}
\end{array}\right) & =\frac{4}{(2 \pi \hbar)^{2}} \int_{\mathbb{R}^{2}}\left(\begin{array}{c}
1 \\
\mathscr{E}(p)
\end{array}\right) w(\mathbf{x}, \mathbf{p}, t) \frac{\partial^{3} \mathscr{E}(p)}{\partial p_{i} \partial p_{j} \partial p_{k}} v_{l} d \mathbf{p}, \\
n(\mathbf{x}, t)\left(\begin{array}{c}
L_{i j k l}^{(0)} \\
L_{i j k l}^{(1)}
\end{array}\right) & =\frac{4}{(2 \pi \hbar)^{2}} \int_{\mathbb{R}^{2}}\left(\begin{array}{c}
1 \\
\mathscr{E}(p)
\end{array}\right) w(\mathbf{x}, \mathbf{p}, t) \frac{\partial^{4} \mathscr{E}(p)}{\partial p_{i} \partial p_{j} \partial p_{k} \partial p_{l}} d \mathbf{p}, \\
C_{W}[w] & =\frac{4}{(2 \pi \hbar)^{2}} \int_{\mathbb{R}^{2}} \mathscr{E}(p) w(\mathbf{x}, \mathbf{p}, t) d \mathbf{p}, \\
C_{V_{i}}[w] & =\frac{4}{(2 \pi \hbar)^{2}} \int_{\mathbb{R}^{2}} v_{i} w(\mathbf{x}, \mathbf{p}, t) d \mathbf{p}, \\
C_{S_{i}}[w] & =\frac{4}{(2 \pi \hbar)^{2}} \int_{\mathbb{R}^{2}} v_{i} \mathscr{E}(p) w(\mathbf{x}, \mathbf{p}, t) d \mathbf{p},
\end{aligned}
$$

that must be expressed as function of the basic variables $n, W, \mathbf{V}, \mathbf{S}$. Therefore the so-called closure problem arises. In the semiclassical case, explicit closure relations have been obtained by resorting to the Maximum Entropy Principle (MEP) [10]. The analogous formulation of MEP for the Wigner function is not straightforward mainly because $w$ is not a probability density function. In [1] a hydrodynamic model has been obtained under the following assumptions (see also [11]):

1. a regime in which quantum effects can be considered only a perturbation of the semiclassical model;

2. the collision term has the same form as in the semiclassical case, an approximation widely discussed in [12]. 
For simplicity we will skip all details and refer the interested reader to [1], but underline some critical point of the theory. For the calculation of the collisional term $C[w]$ the following argumentation is used.

We suppose that the expansion

$$
w=w^{0}+\hbar^{2} w^{(1)}+O\left(\hbar^{4}\right)
$$

holds. By proceeding in a formal way, as $\hbar \rightarrow 0$, the Wigner equation gives the semiclassical Boltzmann equation; therefore we identify $w^{(0)}(\mathbf{x}, \mathbf{p}, t)$ with the semiclassical distribution. As a consequence of (6) the following expansion for the collision term is obtained

$$
C[w] \approx C_{0}\left[w^{(0)}\right]+\hbar^{2} C_{1}\left[w^{(1)}\right]
$$

where $C_{0}\left[w^{(0)}\right]$ is identified with the semiclassical collision operator while, according to [11], the second term is modelled as

$$
C_{1}\left[w^{(1)}\right]=-\frac{1}{\tau}\left(w^{(1)}-w_{e q}^{(1)}\right)
$$

where $w_{e q}^{(1)}$ is the first order term in $\hbar^{2}$ of the equilibrium Wigner function and $1 / \tau$ plays the role of a collision frequency that in general depends on $\mathbf{p}$.

Since, in the limit $\frac{1}{\tau} \rightarrow+\infty$ one formally gets $w^{(1)}=w_{e q}^{(1)}$, we suppose that $w^{(1)}=$ $w_{e q}^{(1)}$, with a good approximation (a similar assumption was made also in [13]). Therefore, it is necessary to find out the expression of $w_{e q}^{(1)}$.

It can be obtained by using the Jaynes approach [14] of maximizing the entropy under suitable constraints on the expectation values, a crucial issue being the expression of the entropy in the quantum case. In $[11,15]$ the standard prescription proposed by von Neumann has been adopted, which leads to a semiclassical limit represented by the Maxwell-Boltzmann distribution. That is, with this assumption, the zero-order term of the equilibrium Wigner function does not coincide with the Fermi-Dirac distribution but gives the Boltzmann-Maxwell low density limit.

To avoid this problem it is needed to consider a different function for the entropy. For this purpose it is also important to observe that, as pointed out in recent papers $[16,17]$, in a closed system the entropy must be conserved because the evolution of the system is described by unitary operators. Instead, in an open system, like a semiconductor electron device, there is a fast decay of the off-diagonal terms and practically only the diagonal contribution survives. Arguing on such a remark, in [17] it has been suggested to use as entropy only the diagonal contribution of the density operator and it has been proved that it increases in time according to the second law of thermodynamics. A suggested expression, already considered in [18], more recently pointed out in [16] and employed in [19], could be the following one

$$
-k_{B} \sum_{k}\left[<\hat{\rho}_{k k}>\log <\hat{\rho}_{k k}>\mp\left(1 \pm<\hat{\rho}_{k k}>\right) \log \left(1 \pm<\hat{\rho}_{k k}>\right)\right]
$$

where the $<\hat{\rho}_{k k}>$ 's are the expectation values of the diagonal elements of the density operator, which can be interpreted as occupation numbers, the upper sign being valid for Bosons and the lower one for Fermions. This could solve the problem of the limit 
of the equilibrium Wigner function, but the needed calculations become much more involved to carry out analytically [16]. We are confident that the quantum correction given in [1] is good enough for determining the quantum extension of the semiclassical hydrodynamic model. These issues are under current investigation [20] and the consequence for the transport theory by the Wigner equation will be the subject of a forthcoming article.

Remark. It is customary in the literature to try to recover some form of Bohm potential. A standard way (see [13]) is that of introducing the approximation

$$
\nabla \log n \approx \beta q \nabla \Phi
$$

into the closure relations. Strictly, such a relation is valid only at equilibrium in the case of low densities. However, for high values of the quasi-Fermi potential $\phi_{F}\left(\phi_{F} \gg\right.$ $k_{B} T$ ), the above approximation has no rationale (see [1]). Therefore, we prefer to avoid the introduction of relation (9).

\section{Energy-transport and drift-diffusion limit models}

It is possible to deduce an energy-transport model from the hydrodynamic one under a suitable scaling. In the semiclassical case, the advantages of the energy-transport formulation are a better regularity of the solutions and the possibility of applying efficient numerical schemes like the Scharfetter-Gummel one [21]. In the presence of quantum corrections we expect also better features of solutions and a better performance of the numerical scheme. If we consider a long time scaling, the evolution equations (4)-(5) tend to the stationary case. In particular, the equations for $n \mathbf{V}$ and $n \mathbf{S}$ become a linear system for these variables

$$
\left(\begin{array}{ll}
c_{11} & c_{12} \\
c_{21} & c_{22}
\end{array}\right)\left(\begin{array}{l}
n V_{i} \\
n S_{i}
\end{array}\right)=\left(\begin{array}{l}
b_{i}^{(0)} \\
b_{i}^{(1)}
\end{array}\right)
$$

where the coefficients $c_{i j}$ depends on the energy $W$ (for explicit expressions see [1]) while

$$
\begin{aligned}
b_{i}^{(0)}= & \frac{\partial}{\partial x_{j}}\left(n(\mathbf{x}, t) F_{i j}^{(0)}-\frac{\hbar^{2}}{24} \frac{\partial^{2}\left(n(\mathbf{x}, t) H_{i j k l}^{(0)}\right)}{\partial x_{k} \partial x_{l}}\right)-q \frac{\partial}{\partial x_{j}} \Phi(\mathbf{x}) n(\mathbf{x}, t) G_{i j}^{(0)} \\
& +\frac{q \hbar^{2}}{24} \frac{\partial^{3} \Phi(\mathbf{x})}{\partial x_{j} \partial x_{k} \partial x_{l}} n(\mathbf{x}, t) L_{i j k l}^{(0)}, \\
b_{i}^{(1)}= & \frac{\partial}{\partial x_{j}}\left(n(\mathbf{x}, t) F_{i j}^{(1)}-\frac{\hbar^{2}}{24} \frac{\partial^{2}\left(n(\mathbf{x}, t) H_{i j k l}^{(1)}\right)}{\partial x_{k} \partial x_{l}}\right)-q \frac{\partial}{\partial x_{j}} \Phi(\mathbf{x}) n(\mathbf{x}, t) G_{i j}^{(1)} \\
& +\frac{q \hbar^{2}}{24} \frac{\partial^{3} \Phi(\mathbf{x})}{\partial x_{j} \partial x_{k} \partial x_{l}} n(\mathbf{x}, t) L_{i j k l}^{(1)} .
\end{aligned}
$$


One gets

$$
\left(\begin{array}{l}
n V_{i} \\
n S_{i}
\end{array}\right)=\frac{1}{c_{11} c_{22}-c_{12} c_{21}}\left(\begin{array}{cr}
c_{22} & -c_{12} \\
-c_{21} & c_{11}
\end{array}\right)\left(\begin{array}{c}
b_{i}^{(0)} \\
b_{i}^{(1)}
\end{array}\right)
$$

and by inserting these relations into the equations for $n$ and $n W$, one obtains the following stationary quantum energy-transport model

$$
\begin{aligned}
& \frac{\partial}{\partial x_{i}}\left[\frac{1}{c_{11} c_{22}-c_{12} c_{21}}\left(c_{22} b_{i}^{(0)}-c_{21} b_{i}^{(1)}\right)-\frac{\hbar^{2}}{24} \frac{\partial^{2}\left(n(\mathbf{x}, t) T_{i j k}^{(0)}\right)}{\partial x_{j} \partial x_{k}}\right]=0, \\
& \frac{\partial}{\partial x_{i}}\left(\frac{1}{c_{11} c_{22}-c_{12} c_{21}}\left(-c_{12} b_{i}^{(0)}+c_{11} b_{i}^{(1)}\right)-\frac{\hbar^{2}}{24} \frac{\partial^{2}\left(n(\mathbf{x}, t) T_{i j k}^{(1)}\right)}{\partial x_{j} \partial x_{k}}\right) \\
& -q \frac{\partial}{\partial x_{i}} \Phi(\mathbf{x}) \cdot n(\mathbf{x}, t) V_{i}+\frac{q \hbar^{2}}{24} \frac{\partial^{3} \Phi(\mathbf{x})}{\partial x_{i} \partial x_{j} \partial x_{k}} n(\mathbf{x}, t) T_{i j k}^{(0)}=C_{W}[w] .
\end{aligned}
$$

Moreover, let us model the energy collision term in the relaxation approximation form

$$
C_{W}[w]=-\frac{W-W_{0}}{\tau_{W}}
$$

with $\tau_{W}$ the energy relaxation time and $W_{0}$ the equilibrium energy. In the limit for the scaled $\tau_{W}$ tending to zero, from equation (12) one formally has $W=W_{0}$ and only the equation (11) remains which is equivalent to the conservation of the total current $\mathbf{J}$, i. e.

$$
J_{i}=\frac{1}{c_{11} c_{22}-c_{12} c_{21}}\left(c_{22} b_{i}^{(0)}-c_{21} b_{i}^{(1)}\right)-\frac{\hbar^{2}}{24} \frac{\partial^{2}\left(n(\mathbf{x}, t) T_{i j k}^{(0)}\right)}{\partial x_{j} \partial x_{k}}=\bar{J}_{i}, i=1,2,(13)
$$

$\overline{J_{i}}$ being constant values. Equation (13) coupled with the Poisson equation for the electrostatic potential constitutes the limiting stationary drift-diffusion model deduced from the energy-transport one. It is a generalization of the standard quantum model based on the Bohm potential (see [22] for a review of the current models known in the literature).

The quantities involving (13) can be explicitly evaluated in terms of the Fermi energy $\mathscr{E}_{F}$ and the electrostatic potential $\Phi$ (we omit the details of the derivation. The interested reader can get them starting from the results in [1]).

Acknowledgements This research was funded by INdAM (GNFM) and from Università degli Studi di Catania, Piano della Ricerca 2020/2022 Linea d'intervento 2 "QICT" and by the project AIM 1893589, Mobilità dei Ricercatori Asse I del PON R\&I 2014-2020. The paper was written within the activity of the Interdipartimental Center for Mathematics in Industry, University of Catania. 


\section{References}

1. L. Luca, V. Romano, Quantum corrected Hydrodynamic Models for Charge Transport in Graphene, Annals of Physics, 406, 30-53 (2019).

2. L. Luca, V. Romano, Comparing linear and nonlinear hydrodynamical models for charge transport in graphene based on the Maximum Entropy Principle, International Journal of Non-Linear Mechanics 104, 39-58 (2018).

3. L. Luca, V. Romano, Hydrodynamical models for charge transport in graphene based on the Maximum Entropy Principle: The case of moments based on energy powers, Atti della Accademia Peloritana dei Pericolanti, 96, No. S1, A5 (2018).

4. V. D. Camiola, and V. Romano, Hydrodynamical model for charge transport in graphene, Journal of Statistical Physics 157 114-1137 (2014).

5. G. Mascali, V. Romano, Charge Transport in graphene including thermal effects, SIAM J. Appl. Mathematics 77, 593-613 (2017).

6. L. Barletti, Hydrodynamic equations for electrons in graphene obtained from the maximum entropy principle, J. Math. Phys. 55(8), 083303, (2014).

7. A. H. Castro Neto, F. Guinea, N. M. R. Peres, K. S. Novoselov, A. K. Geim, The electronic properties of graphene, Rev. Modern Phys. 81, 109 (2009).

8. O. Morandi, F. Schürrer, Wigner model for quantum transport in graphene, J. Phys. A: Math. Theor., 44, 265301 (2011)

9. O. Muscato and W. Wagner, A stochastic algorithm without time discretization error for the Wigner equation, Kin. Rel. Models, 12(1), 59-77, (2019)

10. G. Mascali, V. Romano, Exploitation of the Maximum Entropy Principle in Mathematical Modeling of Charge Transport in Semiconductors, Entropy 19(1), 36 (2017); DOI:10.3390/e19010036 (open access article).

11. V. Romano, Quantum corrections to the semiclassical hydrodynamical model of semiconductors based on the maximum entropy principle, J. Math. Physics 48, 123504 (2007).

12. D. Querlioz, P. Dollfus, The Wigner Monte Carlo Method for Nanoelectronic Devices, ISTE Wiley (2010).

13. C. L. Gardner, The quantum hydrodynamic model for semiconductor devices, SIAM J. Appl. Math. 54 (2), 409 (1994).

14. V.D. Camiola, G. Mascali, V. Romano, Charge Transport in Low Dimensional Semiconductor Structures, Springer, Switzerland (2020) 54 (2), 409-427 (1994).

15. L. Luca, V. Romano, Comparing linear and nonlinear hydrodynamical models for charge transport in graphene based on the Maximum Entropy Principle, Int. J. Non-Linear Mech., 104, 39-58 (2018).

16. D.W. Snoke, G. Liu, S.M. Girvin, The basis of the Second Law of thermodynamics in quantum field theory, Ann. Phys., 327, 1825-1851 (2012).

17. A. Polkovnikov, Microscopic diagonal entropy and its connection to basic thermodynamic relations. Ann. Phys., 326, 486 (2011).

18. E. T. Jaynes, Information Theory and Statistical Mechanics II, Phys. Rev, 108, 171 (1957).

19. L. Barletti, C. Cintolesi, Derivation of Isothermal Quantum Fluid Equations with Fermi-Dirac and Bose-Einstein Statistics, Journal of Statistical Physics 148, 353-386 (2012)

20. V.D. Camiola, L. Luca, V. Romano, Equilibrium Wigner Function for Fermions and Bosons in the Case of a General Energy Dispersion Relation, Entropy, 22, 1023 (2020)

21. V. Romano, 2D numerical simulation of the MEP energy-transport model with a finite difference scheme, J. Comp. Physics 221 439-468 (2007).

22. A. Jüngel, Transport Equations for Semiconductors, Springer-Verlag, Berlin Heidelberg (2009). 